\title{
Type A Behaviours and Heart Disease: Epidemiological and Experimental Foundations
}

\author{
PAUL BENNETT ${ }^{1}$ and DOUGLAS GARROLL ${ }^{2}$ \\ ${ }^{1}$ School of Psychology, University of Wales College of Cardiff, P.O. Box 901, Cardiff, CF1 3rG, \\ Wales \\ ${ }^{2}$ Department of Psychology, Glasgow College, Cowcaddens Road, Glasgow, G4 OBA, Scotland
}

\begin{abstract}
This paper critically examines three strands of evidence that concern the relationship between type A behaviours and coronary heart disease; prospective epidemiological studies of healthy populations, studies of those at high risk for coronary heart disease, and angiographic studies of atherosclerosis. The first of these would seem to provide the strongest test. Methodological and conceptual issues mean that the results of studies using the other methods should be interpreted with care. It is concluded that there is relatively strong evidence of an association between Type A behaviour as measured by Structured Interview and coronary heart disease. Hostility and anger appear to be the most powerful determinants of CHD. However, it is likely that they interact with other type A behaviours and related environmental factors in determining risk.
\end{abstract}

\section{Introduction}

Behavioural factors (diet and cigarette smoking) have long been implicated in the aetiology of coronary heart disease (CHD). However, behavioural influences of this sort are relatively indirect; behaviour acts merely as an intermediary between pathogenic substances and the cardiovascular system. Much more exciting from a psychological perspective, is the prospect that behaviour may exert a more direct influence. Such a notion is not new. Sir William Osler in 1910 described the person most likely to develop angina as "... robust, the vigorous in mind and body, the keen and ambitious man, the indicator of whose engines are always at 'full speed ahead" ". However, it was the research of two American cardiologists, Friedman and Rosenman, in the 1950s which most kindled recent interest. They elaborated the concept of Type A behaviour, proposing a direct link between it and CHD. Type A behaviour was characterized by Friedman and Rosenman (1959), as excessive competitive striving, time urgency and aggressiveness. According to Rosenman (1978a), individuals exhibiting Type A behaviour were displaying "an action-emotion complex in response to their chronic and excessive struggle to achieve more and more from their own environment in too short a period of time, and against the opposing efforts of other persons or things in the same environment." In contrast, Type B behaviour was characterized by the relative absence of such features.

The strength of evidence linking type A behaviour to CHD achieved its nadir with the publication of the Consensus Report (Review Panel, 1981)

$0953-4180 / 90 / 040261+17 \$ 3.50 / 0 \quad$ (C) 1990 GNS (Clinical Neuroscience) Publishers 
where the status of type A behaviour as a risk factor was agreed to be equivalent to that of other traditional risk factors such as hyperlipidaemia and blood pressure. Since then, however, there has been increasing debate as to the validity of this claim. The evidence relating type A behaviour to CHD has become increasingly confused and apparently conflicting. This paper attempts to tease out a number of key issues in an attempt to reduce some of the confusion, account for some of the conflicting evidence, and in doing so reassess the link between type A behaviour and heart disease. Research relating to three key areas of evidence will be assessed: prospective epidemiological studies of healthy populations; studies of populations at high risk of heart disease; studies relating degree of atherosclerosis to type A behaviour.

\section{Prospective Studies in Populations Free From GHD}

The Western Collaborative Group Study (WCGS; Rosenman et al., 1975) provided the first substantive evidence of a link between type A behaviour and CHD. Type A behaviour was measured using the Structured Interview (SI; Rosenman, 1978b) in a population of 3154 male, predominantly white, non-manual workers aged between 39 and 59. All were free of CHD at the beginning of the study, and were followed up for a period of 8.5 years. Over this period, those men assessed as type A were found to have doubled the incidence of CHD than type Bs when all other risk factors were statistically controlled for. Type A behaviour was more strongly linked to angina than myocardial infarction (MI), with relative risks of 2.5 and $2 \cdot 1$ respectively (Matthews and Haynes, 1986).

The WCGS population has now been followed for 22 years (Ragland and Brand, 1988a). By this time 214 men had died from CHD, of whom 119 were type A and 95 type B at baseline. This difference only approached significance. In addition, proportional hazards analyses revealed only a weak association between type A behaviour and CHD mortality at 8.5 years. These negative findings may be considered a serious challenge to the type ACHD hypothesis. However, as Gallacher (1989) notes, mortality from CHD does not equate with incidence of CHD. Proportional hazards analyses revealed type $A$ behaviour to be significantly associated with incidence of CHD, but not mortality, at 8.5 years. Thus, he argues, Ragland and Brand are not presenting data inconsistent with previous findings, but extending the debate to other outcomes. Of interest then is the explanation as to why type A behaviour may be related to incident CHD, but not total mortality from CHD over time. One plausible hypothesis is the potential for behavioural change following the onset of disease. A type A "survivor" may make behavioural changes, and reduce risk of disease progression (Friedman et al., 1986). Such changes are unavailable to type Bs. Ragland and Brand (1989) note the plausibility of such a hypothesis and note also that type As with CHD may modify other risk factors or comply with medical regimes more than their type B counterparts. Long-term incidence studies are clearly required to resolve these issues. 
A total of 2750 subjects free of CHD after 2 years of the WCGS were subsequently assessed using the Jenkins Activity Survey (JAS; Jenkins et al., 1979) a questionnaire derived from the SI. After 4 years 120 subjects who developed CHD were matched with a larger randomly selected group of those that had not. Using a prospective design analysis, those in the upper third of the JAS score distribution were found to have a relative risk of 1.8 for CHD when compared to those in the lowest third (Jenkins et al., 1974). JAS score was not associated with any particular manifestation of CHD (MI, silent MI, angina). When the population was simply dichotomized, and the effect of other risk variables statistically partialled out, the relative risk of the higher JAS group relative to the low dropped to a non-significant value of 1.3 (Brand et al., 1978). Comparison of extremes may be the most appropriate form of analysis in this context, as JAS scores within one standard deviation of the mean show little relationship with SI ratings (Jenkins et al., 1979).

Despite the relative predictive weakness of the JAS, it has been used in a number of further prospective epidemiological studies. DeBacker et al. (1983) used trichotomized data to assess JAS type A ratings in predicting MI or sudden death in a cohort of 2000 disease free men 5 years after initial assessment. A linear relationship was found between total coronary disease and JAS score, with a relative risk between the two extreme groups of 1.9. No multivariate analyses were conducted to control for other risk factors. A second European study (Appels et al., 1984) report data from the KaunasRotterdam Intervention Study in which 3171 men free from CHD, aged between 45 and 59 at intake were followed up after 9.5 years. No relationship between JAS type A score and subsequent coronary event was found.

Unfortunately, only data on approximately $80 \%$ of subjects recruited into the study was available for analysis because of incomplete JAS forms, suggesting the possibility of bias; for example by reducing the number of extreme type As included in the analysis. The study also failed to exploit the most effective method of demonstrating the relative risk of JAS type A behaviour as the ratings were used simply to dichotomize subjects.

A final prospective study using the JAS in a healthy population at baseline was conducted by Cohen and Reed (1985). They followed a representative population of over 2000 male Japanese living in Hawaii over a period of 8 years. No association between type A behaviour and incidence of angina, MI, or degree of atherosclerosis was found on either univariate or multivariate analysis. However, both the prevalence of type A behaviour and incidence of CHD in the study population were low in comparison to typical western populations. The authors also note that high levels of hostility and anger may be more associated with high JAS scores in Western rather than Japanese cultures. In an earlier prevalence study, Cohen et al. (1979), they found that men who were both type A and had adopted a westernised "work ethic" had a relatively high prevalence of CHD, suggesting an interaction between environmental and cultural variables and the health consequences of type A behaviour. 
Three prospective studies have evaluated the predictive validity of a second type A questionnaire, the Bortner Rating Scale (BRS; Bortner, 1969) in relation to CHD. The first of these, the French-Belgian Collaborative Heart Disease Study (1982) followed four cohorts totalling nearly 3000 men initially free from CHD for between 1 month and 5 years. Type A scores were not significantly predictive of CHD in any single cohort. However, when data from all cohorts were combined type A behaviour was found to be a significant predictor of total CHD, MI, and sudden death with a relative risk of total CHD among men in the top quartile relative to the bottom quartile score of $1 \cdot 8$, and for MI and sudden death $1 \cdot 6$. Two UK studies (Johnston et al., 1987; Mann and Brennan, 1987), however, failed to find any relationship between BRS score and later development of CHD despite the large sample size of each study population. Neither study, though, explore the relationship between type A behaviour and angina.

The Framingham study followed a cohort of 580 men and 700 women aged 45-64 on entry for 8 years (Haynes et al., 1980). This study is particularly important as its design allowed the relationship between gender, type A behaviour, and social class in predicting CHD to be investigated. Univariate analyses showed both Framingham Type A Score (FTAS) type A women and men were at increased risk of developing CHD and angina. Sub-group analysis showed these relationships only to hold for women, and white-collar men. Relative risks for total CHD, MI, and angina were $2 \cdot 1$, 1.3 , and 3.6 respectively in women and $2 \cdot 9,2 \cdot 3$, and 1.8 respectively in male non-manual workers (Haynes et al., 1980; Haynes and Matthews, 1988).

Multivariate analyses confirmed the relationship between FTAS type A score in non-manual men and working women, a marginal association $(p=0.07)$ in manually employed men, and (when tension scores were excluded from regression analysis) a significant association in non-working women.

Two post hoc analyses of WCGS data have used component analyses in an attempt to identify the core pathogenic elements within the complex of type A behaviours. Matthews et al. (1977) found seven items, five of which included ratings of anger or hostility, to be significantly related to the later development of CHD. In a separate analysis Chesney, Hecker and Black (1988) also reported five components to be related to CHD incidence: hostility, speaking rate, immediateness, competitiveness, and type A content. As in the Matthews's study, the strongest predictor of CHD was hostility.

The role of hostility as a precursor to CHD has been further highlighted by a number of prospective studies. Barefoot et al. (1983) followed 255 physicians for 25 years following graduation and, after multivariate analysis, found a significant relationship between hostility scores on the CookMedley scale (Cook and Medley, 1954) and later morbidity and mortality from CHD. The CHD incidence density for those scoring above the median was nearly five times greater than those scoring below the median. In a second study of lawyers (Barefoot et al., 1987) those with high hostility scores were again found to have a high risk of developing CHD.

Shekelle et al. (1983) examined mortality rates for CHD during the final 
10 years of a 20 -year follow-up period in a population of 1877 middle-aged men. A weaker, but still significantly enhanced, relative risk of 1.42 was found for those with high hostility scores. The lower risk ratio here may reflect a survival effect. Surviving hostile men may be biologically hardier, while biologically vulnerable hostile men may have died during the early years of follow-up or even prior to the selection of the sample. The relationship between other risk factors (e.g. smoking; Dawber, 1980) and CHD also diminishes with age. Such findings may also account for the failure of Leon et al. (1988) to find a relationship between hostility and CHD in a group of men with baseline measures taken at an average age of 47 years.

Two other studies have failed to find a relationship between hostility and the later development of CHD: McCranie et al. (1986) in a group of 478 physicians after an identical follow-up period to that of Barefoot et al., Hearn et al. (1989) at 33-year follow-up in a sample of 1399 students. The former finding may be attributed to biases in form completion (these were completed at selection interview, and mean hostility scores were considerably lower than those reported by Barefoot $e t$ al.). With regard to the latter, as the authors note, it is possible that the measurement of hostility at the age of 19 does not yield a stable characteristic and accordingly may be subject to change as a result of the experience of college life.

One further theoretical issue is pertinent to all the previous studies. Hostility is not a unitary construct. For example, in a large scale study of the construct validity of the Cook and Medley scale, Smith and Frohm (1985) suggest that the scale measures distrust, suspiciousness, resentment, frequent anger and cynical distrust of others rather than overtly aggressive behaviour. Other measures of hostility (e.g. Buss and Durkee, 1957) equally encompass a number of dimensions. Just as the case of type A behaviour it is possible that included in hostility scales are some items unrelated, to the development of CHD. Some evidence for this results from a re-analysis of the data on law students conducted by Barefoot $e t$ al. (1989). They derived five sub-scales for the Cook and Medley scale, three of which (cynicism, aggressive responding and hostile affect) predicted mortality more strongly than the total score.

\section{Comment}

A majority of prospective studies support the notion of type A behaviour being an independent predictor of CHD, particularly for those working in an environment that may be considered likely to elicit such behaviours (e.g. Rosenman et al., 1975; Haynes et al., 1980; Cohen et al., 1979). The strength of the link would appear to depend on the nature of the assessment used. However, only for the BRS do the negative findings outweigh the positives.

Many studies identify a univariate link between type A behaviour and CHD, which becomes non significant when other factors are partialled out. From such outcomes it is impossible to conclude direct causal links between type A behaviour. However, some caution should be exercised here. The 
relationship between type A behaviour and CHD, in some cases, may indeed be indirect (for example through the higher prevalence of smoking or consumption of alcohol among type As). However, we should be careful not to overlook the possibility of causal pathways through which type A behaviour affects on primary risk factors. For example type A behaviour may exert an influence through effects on biological risk factors such as serum cholesterol levels (e.g. Rosenman et al., 1975). While mechanisms such as this weakens the case for type A behaviour as an independent risk factor, they do locate the behaviour within a causal chain.

One further methodological issue should be raised. One disease endpoint often disregarded in epidemiological studies is that of angina. This neglect may serve to underestimate the aetiological role of type A behaviour. Type A behaviour may result in transient increases in blood pressure, excessive demands on cardiac output, and cardiac ischaemia (angina) in those with some degree of coronary artery disease. Preliminary evidence, for example reported by Young et al. (1980), indicates a closer relation between type A behaviour and a history of angina than with atherosclerosis.

Of the type A behaviours that appear linked to the development of CHD, hostility would seem the most pathogenic. However, it must also be conceded that hostility is not the sole precursor of heart disease. Reported type A behaviour, speech characteristics during SI, and competitiveness are all to some extent potentially implicated, and there may be a complex interaction between these various type A behaviours and environmental and cultural factors which predict final risk.

\section{Prospective Studies of "High Risk" Individuals}

A second strand of evidence linking type A behaviour (and other risk factors) to CHD stems from prospective studies of those identified to be at high risk of developing CHD. Studies of this sort have the advantage of a relatively high percentage of subjects developing CHD (therefore reducing the required sample size), but have the serious disadvantage that they are no longer based on representative populations. This undoubtedly restricts the interpretation of their results.

In the Multiple Risk Factor Intervention Trial (MRFIT; Shekelle et al., $1985 a$ ) the SI was administered to 3110 men aged $35-57$ years within the top $10 \%$ of risk distribution for CHD. Care was taken to ensure high quality control of the interviews and interview ratings (see Scherwitz, 1988). After initial assessment subjects were allocated to either usual care or special counselling. SI-defined type A was not associated with risk of coronary death, MI, or total mortality in either the usual care or special intervention groups after controlling for other potentially confounding factors at an average of seven years follow up.

These data have been viewed as particularly challenging to the coronary-prone hypothesis and Shekelle and his colleagues have defended their results against a number of attacks. They argue that their sample size was 
sufficiently large to achieve adequate statistical power although Haynes and Matthews (1988) has calculated that the power to detect a statistical association in the usual care group was low $(0 \cdot 67)$. Shekelle et al. also note that type A behaviour has previously been predictive of CHD amongst individuals at high risk due to other risks factors (Haynes et al., 1983).

Differences in the style of SI administration and scoring between the WCGS and the MRFIT team may also have resulted in some misclassification of subjects in the latter, but as Scherwitz (1988) argues if the interview assessment is not sufficiently robust to transfer between studies, its validity as a measure of type A behaviour must be strongly questioned. Further evidence of the pathological status of hostility, however, can be derived from a re-analysis of the MRFIT data conducted by Dembroski $e t$ al. (1989). They compared CHD cases with matched controls on componential analyses of SI ratings. Measures of potential for hostility and stylistic hostility were both strongly associated with CHD incidence after adjustment for their risk factors in younger (age less than 47 years at baseline) participants.

The first study to follow post-infarction patients (Jenkins et al., 1976) was also one of the few to report a positive relationship between type A behaviour and the recurrence of disease. Using the JAS as a measure of type A behaviour, 267 men who had at least one coronary heart disease event either during or prior to the WCGS baseline survey were followed for at least one year. Stepwise discriminant function analyses showed the JAS score to be a significant predictor of recurrent MI after controlling for other risk factors.

Three later prospective studies based on JAS scores have, however, failed to replicate these findings. Dimsdale et al. (1981) studied predictors of further disease developments (e.g. MI, death, hospitalization) in 189 men following cardiac catheterization over a period of one year. Stepwise discriminant function analyses showed that if anything, type B behaviour was predictive of new morbidity. Shekelle et al. (1985b) investigated the predictors of recurrent myocardial infarction in 2314 participants in a trial examining the effectiveness of aspirin in reducing subsequent risk of reinfarction. All subjects were given the JAS on entry and were followed for minimum of three years. Type A was unrelated to re-infarction.

In a third study, Case et al. (1985) asked 866 patients to complete the JAS within 2 weeks of discharge from a coronary care unit. Five hundred and sixteen did so, and were followed for between 1 and 3 years. No relationship was found between type A score and total and cardiac mortality during this time. However, the validity of responses to a behavioural rating scale given so quickly after a major adverse life event must be questioned. Perhaps even more critical was the high non-response rate and the significantly higher cardiac mortality rate of the non-responders when compared to those subjects entered into the trial $(14.9 \%$ versus $9.5 \% ; p<0.001)$. It is possible that time urgent, hostile, type As refuse to participate in the trial and thus skewed the study population and the results towards a negative finding.

Ragland and Brand (1988) followed 257 men who developed CHD 
during the initial 8.5 year phase of the WCGS for an average of 13 years. Again, type $B$ behaviour was associated with significantly greater risks of reinfarction than type A behaviour (mortality rate for type A subjects $=19 \cdot 1$ per 1000 person years; for $B s=31 \cdot 7$ per 1000 person years). The authors speculate that type As may have been more compliant to medical regimes than type Bs or sought medical help earlier and therefore may have had a lower disease severity. They also note, although discount, the argument that type As may have changed their behaviour and therefore reduced their risk (but see Gallacher, 1989; Ragland and Brand, 1988). It must be remembered that the SI was administered on average 4.5 years before the coronary event; no assessment of behaviour was made after the event.

Powell and Thoresen (1985) did measure type A behaviour a minimum of 6 months following MI. Subjects, who were either treatment or control cases in the Recurrent Coronary Prevention Project were administered the Videotaped Structured Interview (VSI; Friedman and Powell, 1984) on entry to the study. Those that had a recurrent cardiac event within the first 2 years of the trial were matched to a control group on measures of traditional risk factors. After controlling for treatment status (but not behavioural change), 15 items relating to type A behaviour were found to have a univariate association with recurrent events. Three type A behaviours (emotional intensity, self-involvement, and reported arousal whilst driving) had a multivariate relationship.

\section{Comment}

A number of methodological and interpretive issues are raised by studies of post-infarction patients. Pickering (1985a) argues that all such studies are able to demonstrate by this methodology is that the major risk factors after MI are not the same as those before MI. He cites the results from the Multicenter Postinfarction Research Group (1983) study in which serum cholesterol level, blood pressure, and smoking were not predictive of survival after MI. If these are not consistently predictive, he argues, "it is hardly surprising that the role of type A behaviour also cannot be demonstrated". Examination of regression analyses from all the cited studies (where reported) reveals no consistent support for any one of the classic risk factors with regard to re-infarction.

Why, then did type A behaviour apparently confer a protective effect in the Ragland and Brand paper? Analysis of the contributors to risk revealed low relative hazards of re-infarction attributable to smoking, systolic blood pressure and smoking. Changes in these risk factors therefore can be considered to exert little influence on re-infarction. However, it is possible that the apparent protective effect of type A behaviour reflects its measurement on average 4.5 years before the event. Type A behaviour may have contributed to the first event, but changes in behaviour (i.e. reductions in type A behaviour whether as a result of disease, treatment with beta-blockers, or self-managed change) may have conferred a later protective effect. It is a failing of all the cited studies (with the exception of Powell and Thoresen) 
that they take as a measure the behaviour before the onset of disease, thus failing to take into account the potential for subsequent behavioural change.

\section{Modification of Type A's Behaviours}

Changes in type A behaviour as a mediator of post-infarction risk have been speculated upon already as preventive against disease progression. One study, the Recurrent Coronary Prevention Project (RCCP; Friedman et al., 1986) has examined this issue in some detail.

They compared the effectiveness of group cardiac counselling versus cardiac counselling with type A behaviour modification in over 800 subjects who had an infarction at least 6 months previously and who did not smoke. Cardiac counselling consisted primarily of discussions aimed at increasing adherence to prescribed dietary, exercise and drug regimes. Type A counselling used a wide variety of cognitive and behavioural techniques to modify type A behaviour.

By the final assessment, at 4.5 years, cumulative total re-infarction was virtually halved in the combined treatment group $(12.9 \%$ versus $21.2 \%)$ and death from CHD was significantly reduced $(5 \cdot 2 \%$ versus $7 \cdot 2 \%)$ when compared to cardiac counselling alone. Perhaps even more persuasively, participants in either group who reported significant behavioural change at the end of the first year subsequently had a significantly lower cumulative recurrence rate $(6.6 \%$ versus $17.2 \%)$ in the remaining 3.5 years than those that failed to do so.

\section{Atherosclerosis Studies}

One apparently simple procedure by which to assess the impact of type A behaviour on CHD is to correlate the degree of type A behaviour with the extent of atherosclerosis during routine angiography. It should be noted that this assumes one causal mechanism for the pathogenic process linked to type A behaviour. It ignores one of the most common causes of CHD death or angina, cardiac arrhythmias and vaso-spasm, limiting any conclusions that may be drawn. For example, Young et al. (1980) found cholesterol and smoking history were more closely associated with degree of atherosclerosis, whilst hypertension and type A behaviour (measured on a modified JAS) were more related to a history of angina.

In a series of studies conducted at Duke University (Blumenthal et al., 1978; Williams et al., 1980; Blumenthal et al., 1987) degree of type A behaviour has been found to correlate positively with the severity of atherosclerosis. Other, independent, studies have replicated these findings (Krantz et al., 1979; Seeman and Syme, 1987; Frank et al., 1978).

However, a number of studies using the SI have found no relationship between type A behaviour and atherosclerosis. Dimsdale et al. (1979) found no relationship between SI ratings of type A behaviour and atherosclerosis, even when extremes of type A behaviour only were used in the analysis. 
Krantz et al. (1981) and Scherwitz et al. (1983) also failed to find an association between SI rated type $A$ behaviour and atherosclerosis. In component analysis studies, Dembroski et al. (1985) and MacDougall et al. (1985) also found no relationship between global measures of type A behaviour and atherosclerosis. As the latter studies were essentially reanalyses of previously reported data they cannot be considered as further independent negative findings.

One of the serious problems with the SI data stems from an over representation of type As without underlying coronary artery disease (type A behaviour is related to chest pain independently of degree of atherosclerosis; Pearson, 1984) amongst angiography patients. This influences the validity of the relative risk estimates when the disease rates in the study groups are also unrepresentative of the population (Haynes and Matthews, 1988). This problem is exacerbated by generally small sample sizes in these studies. It is perhaps worth noting that those studies which report an association between type $\mathrm{A}$ behaviour and stenosis have, on average, larger samples and a smaller percentage of type A subjects. A further problem for angiographic studies based on SI ratings is that treatment with beta-blockers which are known to affect speech characteristics and other behaviours upon which ratings are made (e.g. Krantz et al., 1982), and are in common use at the time of angiography (Pickering, 1985b), may result in an under reporting of type A behaviour.

A substantial study of nearly 2300 subjects reported by Williams et al. (1988) provides the strongest evidence of a type A-atherosclerosis relationship and identifies further methodological problems that may have reduced the power of the previous studies to find such a relationship. They found type A behaviour to be significantly associated with degree of atherosclerosis after age, sex, hyperlipidaemia, smoking, hypertension and their various interactions had been controlled for. However, the relationship held only for younger patients, aged 45 or younger, and the effect was lesser than for smoking or hyperlipidaemia. Over this age, there was a trend towards increased severity of coronary artery disease amongst type Bs (a similar diminution of risk with aging was found for other risk variables such as smoking). This reversal of risk may again be due to survival effects. These findings suggest that previous negative findings may be attributable to small sample sizes and the confounding of age with type A behaviour.

Three studies to use both SI and JAS found an association between SI but not JAS type A scores and degree of atherosclerosis (Blumenthal et al., 1978; Krantz et al., 1979; Williams et al., 1988). Dimsdale et al. (1979) also failed to find an association between JAS scores and atherosclerosis even when the comparison was between extreme scores. Zyzanski et al. (1976) report the only positive association between JAS score and degree of atherosclerosis. However, Krantz et al. (1979) did find that extreme JAS type A scores to predicted progression of atherosclerosis between repeat angiograms over a 2-year period.

Studies using the BRS have been unable to find an association between type A behaviour and degree of atherosclerosis (Kornitzer et al., 1982; 
Pearson, 1983) despite the large sample size $(n=792)$ of the latter study. Bass and Wade (1982) actually found that male patients with an obstruction in at least one vessel of greater than $50 \%$ had lower BRS scores than those with no obstruction. No association was found amongst female subjects, although subject numbers were small $(n=32)$.

Both Dembroski et al. (1985) and MacDougall et al. (1985) used SIderived type A component scores as their predictor variable. In both studies, potential for hostility and anger-in were related to degree of atherosclerosis. In the study by Dembroski et al. increases in pathology were only related to hostility in those patients who held their anger-in and a stronger relationship between hostility and atherosclerosis was found amongst younger subjects (Williams et al., 1988). In the second study (MacDougall et al., 1985) although anger-in and hostility were both related to the severity of atheroma, there was no interaction between the two factors. Dembroski and Costa (1988) have since argued that this style of holding anger-in may be a disease consequence rather than cause. Hostility and anger-in have been found to be negatively correlated in healthy populations (e.g. Musante et al., 1983), yet in patients undergoing angiography the overall correlations were near zero. In addition, in those patients with high disease severity the relationship between hostility and anger-in was found to be positive, whilst for those with low levels of atherosclerosis and symptoms this relationship was reversed. Thus, it is possible that anger-out may actually have the aetiological role and the reported associations between anger-in and atherosclerosis result from individuals' attempts to modify their anger response as a result of disease.

Questionnaire methods have also been used to examine the impact of hostility on degree of atherosclerosis. Williams et al. (1980) found the Cook and Medley hostility scale to be more strongly associated with degree of atherosclerosis than global type A SI ratings. In a study by Siegman et al. (1987) two factors representing neurotic hostility and expressive (nonneurotic) hostility were derived from the Buss-Durkee Hostility Inventory (Buss and Durkee, 1957). Neurotic hostility was found to be significantly inversely associated with severity of coronary artery disease, whilst nonneurotic hostility scores were positively associated with the extent of disease. Overall hostility score was unrelated to the extent of atherosclerosis.

\section{Comment}

While evidence of a relationship between SI type A ratings and degree of atherosclerosis is, to an extent, mixed the preponderance of studies (and one substantial study) are supportive of a positive association. Nevertheless, the relationship with type A behaviour is not as strong as that for other risk factors such as smoking and hyperlipidaemia and it seems to hold for younger rather than older subjects; in this, however, it is not unique. The two component studies both suggest hostility and the expression of anger are more closely related to atherosclerosis development than global type A behaviour. Those studies which employed self-completion questionnaire 
methods of assessment have shown little evidence of any relationship, but re-analysis by age may prove fruitful.

Again, it is necessary to voice some reservations about the appropriateness of angiography as a means of assessing the relationship between type $\mathrm{A}$ behaviours and CHD. Pickering (1985b) notes that similar problems of interpretation exist in angiographic studies as in the "high risk" prospective studies. In contrast to strong epidemiological evidence, angiographic studies have equally failed to find a consistent relationship between smoking or blood pressure and extent of atherosclerosis on angiogram (Kornitzer et al., 1982; Hartz et al., 1982; Anderson et al., 1978; Frank et al., 1978).

Other difficulties undoubtedly stem from the different methodologies used in the various studies. Some assess type A behaviour before angiography, some after (with a number of possible biases according to time of assessment), some used the number of vessels occluded by $50 \%$ as their criterion for disease status, some $75 \%$, whilst other have employed more complex indexes of disease status. However, no pattern emerges from the studies to suggest these factors have contributed to a consistent bias. More problematic is the population being examined. The high preponderance of type As and of moderate to severe disease necessarily reduces the likelihood of finding a positive correlation between the two indices because of the low variance in each variable.

\section{Conclusions}

If one presumes a hierarchy of the methodological strength of the various approaches to examining the relationship between type A behaviour and CHD, prospective studies that follow-up healthy subjects at baseline appear the strongest. Evidence from angiographic and "high risk" epidemiological studies adds to the picture, but should be accorded less weight. Further, these latter methods have frequently failed to find consistent relationships between other traditional risk factors such as smoking, serum cholesterol and blood pressure and markers of CHD. It seems inappropriate to question type A behaviour as a risk factor for CHD on the basis of such studies without similarly calling into question other, more traditional, risk factors.

With these reservations in mind, the prevailing evidence would seem to strongly link global type A behaviour as measured by the SI or VSI and the development of CHD. Substantial support emerges from epidemiological, intervention, and angiography methodologies, and arguably, also from the high risk prospective and intervention studies. This probably reflects the fact that these measures of type $\mathrm{A}$ include ratings of hostility and anger potential in determining the global A type assessment. In addition, their ability to detect behaviours that are linked to the development of CHD, such as aggressive speech characteristics may also be crucial.

The original epidemiological evidence (Rosenman et al., 1975) was based on a group of predominantly non-manual workers, and it may be that populations such as this may encounter more situations likely to provoke type A behaviours, have more control over their working environment, and 
more opportunities to seek challenging stimuli (Byrne, 1981; Smith, 1989). Accordingly, type A behaviour, measured at a point in time, may have more circumstantial relevance to non-manual populations.

Support for this argument can be derived from the Framingham study where type A non-manual men and women in work had the highest levels of risk and the study of Japanese men where an interaction between behavioural style and cultural values was found. Thus, type A behaviour must not be considered a stable undifferentiated response to all stimuli. Type A behaviour is elicited by environmental stimuli (Rosenman, 1978), and type As may in turn seek and elicit such stimuli; type A behaviour is perhaps best regarded as a dynamic person-environment transaction (Smith, 1989) and not as a static trait.

Within the complex of type A behaviours, some are more pathogenic than others-some may even be protective. Component analyses and questionnaire based studies have consistently shown hostility and anger to have a close relationship with the development of CHD. Other components of type A behaviour, including competitiveness and various speech characteristics, have also been implicated as precursors of CHD. There may be interactions between these, and other characteristics (e.g. self involvement; Scherwitz et al., 1983) that ultimately determine risk. For example, people who are time urgent may be sensitized to situations in which they are held up, to which they respond with anger. Further, the pathogenic significance of anger and the preferred directional style of expression may depend on the precise demands of anger instigating circumstances (Engebretson et al., 1989).

A consistent problem throughout the type A research, and now work on hostility, is that the measures used are acknowledged as being imperfect, but are used as no suitable alternatives are available Even the SI, the gold standard of all measures of type A behaviour, is suffering increasing problems of drift from its original administration and scoring systems (Bennett and Carroll, 1989; Scherwitz, 1988). No pencil and paper questionnaire of type A measures hostility or anger. We have advanced conceptually-now we should advance methodologically. New measures should be derived specifically to measure key pathogenic components such as overt or cynical hostility, anger, the relevant psychomotor behaviours and so on, according to our new conceptualisations of these behaviours. These should be both trait and state measures to allow consistencies and change over time or situation to be measured (Bennett et al., 1990). Such measures need to be experimentally or prospectively validated, with measures of moderator cultural and environmental variable considered. Such research should contribute to a more accurate determination of the relationship between behavioural style and CHD.

\section{References}

Anderson, A. J., Barboriak, J.J. and Rimm, A. (1978). Risk factors and angiographically determined coronary occlusion. American Fournal of Epidemiology, 107, 8-14. 
Appels, A., Mulder, P. and van't Hof, M. (1984). The predictive power of the A/B typology in Holland. Results of a 10-year follow up study. Paper presented at the conference, Biobehavioral factors in Coronary Heart Disease, Winterscheid.

Barefoot, J. C., Dahlstrom, J. W. and Williams, R. B. (1983). Hostility, CHD incidence, and total mortality: a 25 -year follow-up study of 255 physicians. Psychosomatic Medicine, 45, 59-63.

Barefoot, J. C., Williams, R. B., Dahlstrom, W. G. and Dodge, K. A. (1987). Predicting mortality from scores on the Cook-Medley Scale: A follow-up of 118 lawyers. Psychosomatic Medicine, 49, 210.

Barefoot, J. C., Dodge, K. A., Peterson, B. L., Dahlstrom, W. G. and Williams, R. B. (1989). The Cook-Medley hostility scale: item content and ability to predict survival. Psychosomatic Medicine, 51, 46-57.

Bass, C. and Wade, C. (1982). Type A behaviour not specifically pathogenic. Lancet, 2, $1147-$ 1149 .

Bennett, P. and Carroll, D. (1989). The assessment of type A behaviour: a critique. Psychology and Health, 3, 183--194.

Bennett, P., Gallacher, J. and Johnston, D. (1990). Towards a state measure of type A behaviour. British Journal of Clinical Psychology, 29, 155-165.

Blumenthal, J. A., Kong, Y., Schanberg, S. M., Thompson, L. W. and Williams, R. B. (1978). Type A behavior and coronary atherosclerosis. Circulation, 58, 634-639.

Blumenthal, J. A., Burg, M. M., Barefoot, J., Williams, R. B., Haney, T. and Zimet, G. (1987). Social support, type A behavior, and coronary artery disease. Psychosomatic Medicine, 49, 331-340.

Bortner, R. W. (1969). A short rating scale as a potential measure of pattern A behavior. Journal of Chronic Diseases, 22, 87-91.

Brand, R. J., Jenkins, C. D., Rosenman, R. H. (1978). Comparison of coronary heart disease prediction of the Western Collaborative Group Study using the Structured Interview and the Jenkins Activity Survey assessments of the coronary-prone type A behavior pattern (abstract). American Heart Association Cardiovascular Disease Epidemiology Newsletter, 24.

Buss, A. H. and Durkee, A. (1957). An inventory for assessing different kinds of hostility. Journal of Consulting Psychology, 21, 343-349.

Byrne, D. G. (1981). Type A behaviour, life events and myocardial infarction: independent or related risk factors? British Journal of Medical Psychology, 54, 371-377.

Case, R.B., Heller, S. S., Case, N. B., Moss, A.J. and the Multicenter Post-infarction Research Group (1985). Type A behavior and survival after acute myocardial infarction. Nerw England Fournal of Medicine, 312, 737-741.

Chesney, M. A., Hecker, M. H. L. and Black, G. W. (1989). Coronary-prone components of type A behavior in the WCGS: a new methodology. In "Type A Behavior Pattern. Research, Theory and Intervention" (Eds. B. K. Houston and C. R. Snyder). Wiley, New York.

Cohen, J. B. and Reed, D. (1985). The type A behavior pattern and coronary heart disease among Japanese men in Hawaii. Journal of Behavioral Medicine, 8, 343-352.

Cohen, J B., Syme, S. L., Jenkins, C. D., Kagan, A. and Zyzanski, S. J. (1979). Cultural context of type A behavior and risk for CHD: a study of Japanese American males. Journal of Behavioral Medicine, 2, 375-384.

Cook, W. W. and Medley, D. M. (1954). Proposed hostility and pharasaic-virtue scales for the MMPI. Journal of Applied Psychology, 38, 414-418.

Dawber, T. R. (1980). "The Framingham Study: the Epidemiology of Atherosclerotic Disease." Harvard University Press, Cambridge, MA.

DeBacker, G., Kornitzer, M., Kittel, F. and Dramaix, M. (1983). Behavior, stress and psychosocial traits as risk factors. Preventive Medicine, 12, 32-36.

Dembroski, T. M. and Costa, P. T. (1988) Assessment of coronary prone behavior: a current overview. Annals of Behavioral Medicine, 10, 60-70.

Dembroski, T. M., MacDougall, J. M., Williams, R. B., Heney, T. L. and Blumenthal, J. A. (1985). Components of type A, hostility, and anger-in: relationships to angiographic findings. Psychosomatic Medicine, 47, 219-233. 
Dimsdale, J. E., Hackett, T. P., Catanzano, D. and White, P. J. (1979). The relationship between diverse measures for type A personality and coronary angiographic findings. Journal of Psychosomatic Research, 23, 289-293.

Dimsdale, J. E., Block, P. C., Gilbert, J., Hackett, T. P. and Hutter, A. M. (1981). Predicting cardiac morbidity based on risk factors and coronary angiographic findings. American Journal of Cardiology, 47, 73-76.

Engebretson, T. O., Matthews, K. A. and Scheier, M. F. (1989). Relations between anger expression and cardiovascular reactivity: reconciling inconsistent findings through a matching hypothesis. Fournal of Personality and Social Psychology, 57, 513-521.

Frank, K. A., Heller, S. S., Kornfeld, D. S., Sporn, A. A. and Weiss, M. B. (1978). Type A behavior and coronary angiographic findings. Journal of American Medical Association, 240, $761-763$.

French-Belgian Collaborative Group (1982). Ischemic heart disease and psychological patterns. Prevalence and incidence studies in Belgium and France. Advances in Cardiology, 29, 25-31.

Friedman, M. and Powell, L. H. (1984). Diagnosis and quantative assessment of type A behavior: introduction and description of the Videotaped Structured Interview. Integrative Psychiatry, 2, 123-129.

Friedman, M. and Rosenman, R. (1959). Association of specific overt behavior pattern with blood and cardiovascular findings. Fournal of the American Medical Association, 169, 12861296.

Friedman, M., Thoresen, C. E., Gill, J.J., Ulmer, D., Powell, L. H., Price, V. A., Brown, B., Thompson, L., Rabin, D. D., Breall, W. S., Bourg, E., Levy, R. and Dixon, T. (1986). Alteration of type A behavior and its effect on cardiac recurrences in postmyocardial infarction patients: summary results of the recurrent coronary prevention project. American Heart Fournal, 112, 653-665.

Hartz, A. J., Barboriak, P. N., Anderson, A. J., Hoffman, R. G. and Barboriak, J. J. (1981). Smoking, coronary artery occlusion, and nonfatal myocardial infarction. Fournal of the American Medical Association, 246, 851-853.

Haynes, S G. and Matthews, K. A. (1988). The association of type A behavior with cardiovascular disease---update and critical review. In "Type A Behavior Pattern. Research, Theory and Intervention" (Eds. B. K. Houston and C. R. Snyder). Wiley, New York.

Haynes, S. G., Feinleb, M. and Eaker, E. D. (1983). Type A behavior and the ten-year incidence of coronary heart disease in the Framingham Heart Study. In "Psychosomatic Risk Factors and Coronary Heart Disease" (Ed. R. H. Rosenman). Hans Huber, Berne.

Haynes, S. G., Feinleib, M. and Kannel, W. B. (1980). The relationship of psychosocial factors to coronary heart disease in the Framingham study: III. Eight-year incidence of coronary heart disease. American Fournal of Epidemiology, 111 37-58.

Hearn, M. D., Murray, D. M. and Leupker, R. V. (1989). Hostility, coronary heart disease, and total mortality: a 33-year follow-up study of university students. Fournal of Behavioral Medicine, 12, 105--121.

Jenkins, C. D., Rosenman, R. H. and Zyzanski, S. J. (1974). Prediction of clinical coronary heart disease by a test for the coronary prone behaviour pattern. New England Journal of Medicine, 23, 2171-1275.

Jenkins, C. D., Rosenman, R. H. and Zyzanski, SJ. (1976). Risk of new myocardial infarction in middle-aged men with manifest coronary heart disease. Circulation, 53, 342-347.

Jenkins, C. D., Zyzanski, S. J. and Rosenman, R. H. (1979). "Jenkins Activity Survey Manual." Psychological Corporation, New York.

Johnston, D. W., Cook, D. G. and Shaper, A. G. (1987). Type A behaviour and ischaemic heart disease in middle aged British men. British Medical fournal, 295, 86-89.

Kornitzer, M., Magotteau, V., Degre, C., Kittel, F., Struyven, J. and van Thiele, E. (1982). Angiographic findings and the type A pattern assessed by means of the Bortner scale. Fournal of Behavioral Medicine, 5, 313-320.

Krantz, D. S., Matthews, K. A., Sanmarco, M. E. and Selvester, R. H. (1979). Psychological correlate of progression of atherosclerosis in men. Psychosomatic Medicine, 41, 467-475. 
Krantz, D. S., Davia, J.E., Dembroski, T. M., MacDougall, J. M., Shaffer, R.T. and Schaeffer, M. A. (1981). Extent of coronary atherosclerosis, Type A behavior, and cardiovascular response to social interaction. Psychophysiology, 18, 654 664.

Krantz, D. S., Durel, L. A., Daria, J. E., Shaffer, R. T., Arabian, J. M., Dembroski, T. M. and MacDougall, J. M. (1982). Propanolol medication among coronary patients: relationship to type A behavior and cardiovascular response. Fournal of Human Stress, 8 , 4-12.

Leon, G. R., Finn, S.E., Murray, D. and Bailey, J. M. (1988). The inability to predict cardiovascular disease from hostility scores or MMPI items related to type A behavior. Journal of Consulting and Clinical Psychology, 56, 597--600.

MacDougall, J. M., Dembroski, T. M., Dimsdale, J. L. and Hackett, T. P. (1985). Components of type A, hostility, and anger-in: further relationships to angiographic findings. Health Psychology, 4, 137-152.

Mann, A.H. and Brennan, P.J. (1987). Type A behaviour score and the incidence of cardiovascular disease: a failure to replicate the claimed associations. Fournal of Psychosomatic Research, 31, 685-692.

Matthews, K. A. and Haynes, S. G. (1986). Type A behavior pattern and coronary heart disease risk. American Fournal of Epidemiology, 123, 923-960.

Matthews, K. A., Glass, D. C., Rosenman, R. H. and Borner, R. W. (1977). Competitive drive, Pattern A, and coronary heart disease: a further analysis of some data from the Western Collaborative Group Study. Journal of Chronic Disease, 30, 923-960.

McCranie, E. W., Watkins, L. O., Brandsma, J. M. and Sisson, B. D. (1986). Hostility, coronary heart disease (CHD) incidence, and total mortality: lack of association in a 25year follow-up study of 478 physicians. Fournal of Behavioral Medicine, 9, 119-125.

Osler, W. (1910). The Lumleian Lectures on angina pectoria. Lancet, 1, 839-844.

Pearson, T. A. (1983). Risk factors for arteriographically defined coronary artery disease. Doctoral dissertation from the Johns Hopkins University. 83-16989. University Microfilms International, Ann Arbor, MI.

Pearson, T. A. (1984). Coronary arteriography in the study of the epidemiology of coronary artery disease. Epidemiologic Reviews, 6, 140-166.

Pickering, T.G. (1985a). Should studies of patients undergoing coronary angiography be used to evaluate the role of behavioral risk factors for coronary heart disease? Fournal of Behavioral Medicine, 8, 203-213.

Pickering, T. G. (1985b). Type A behavior and survival after myocardial infarction (letter). New England Fournal of Medicine, 313, 450.

Powell, L. H. and Thoresen, C. E. (1985). Behavioral and physiologic determinants of longterm prognosis after myocardial infarction. Journal of Chronic Diseases, 38, 253-263.

Ragland, D. R. and Brand, R. J. (1988). Type A behavior and mortality from coronary heart disease. New England Journal of Medicine, 318, 65-69.

Review Panel (1981). Coronary-prone behavior and coronary heart disease: a critical review. Circulation, 63, 1199-1215.

Rosenman, R. H. (1978a). The role of Type A behaviour pattern in ischaemic heart disease: modification of its effects by beta-blocking agents. British Fournal of Clinical Practice, 32 (Suppl. 1).

Rosenman, R.H. (1978b). The interview method of assessment of the coronary-prone behavior pattern. In "Coronary-Prone Behavior" (Eds. T. M. Dembroski, S. M. Weiss, J. L. Shields, S. G. Haynes and M. Feinleib). Springer-Verlag, New York.

Rosenman, R. H., Brand, R.J., Jenkins, C. D., Friedman, M., Straus, R., and Wurm, M. (1975). Coronary heart disease in the Western Collaborative Group Study: Final followup experience of $8 \frac{1}{2}$ years. Fournal of the American Medican Association, 233, 872-877.

Scherwitz, L. (1988). Interviewer behaviors in the Western Collaborative Group Study and Multiple Risk Factor Intervention Trial Structure Interviews. In "Type A Behavior Pattern. Research, Theory, and Intervention". B. K. Houston and C. R. Snyder (Eds.). Wiley, New York.

Scherwitz, L., McKelvian, R., Laman, C., Patterson, J., Dutton, L., Yusim, S., Lester, J., Kraft, I., Rochelle, D. and Leachman, R. (1983). Type A behavior, self-involvement, and coronary atherosclerosis. Psychosomatic Medicine, 45, 47-57. 
Seeman, T.E. and Syme, S. L. (1987). Social networks and coronary artery disease: a comparison of the structure and function of social relations as predictors of disease. Psychosomatic Medicine, 49, 341--354.

Shekelle, R. B., Gale, M., Ostfeld, A. M. and Paul, O. (1983). Hostility, risk of coronary disease, and mortality. Psychosomatic Medicine, 45, 219-228.

Shekelle, R. B., Billings, J. H., Borhani, N. O., Gerace, T. A., Hulley, S. B., Jacobs, D. R., Lasser, N. L., Mittelmark, M. B., Neaton, J. D. and Stamler, J. (1985a). The MRFIT behavior pattern study: II. Type A behavior and incidence of coronary heart disease. American Fournal of Epidemiology, 122, 559-570.

Shekelle, R. B., Gale, M. and Norusis, M. for the Aspirin Myocardial Infarction Study Research Group (1985b). Type A score (Jenkins Activity Survey) and risk of recurrent coronary heart disease in the Aspirin Myocardial Infarction Study. American Journal of Cardiology, 56, 221-225.

Smith, T. W. (1989). Interactions, transactions, and the type A behavior pattern: additional avenues for the search for coronary-prone behavior. In "Search of Coronary-Prone Behavior: Beyond Type A" (Eds. A. W. Siegman and T. M. Dembroski). Lawrence Erlbaum, Hillsdale, NJ.

Smith, T. W. and Frohm, K. D. (1985). What's so unhealthy about hostility? Construct validity and psychosocial correlates of the Cook and Medley Ho scale. Health Psychology, 4, 503-520.

Siegman, A. W., Dembroski, T. M. and Ringel, N. (1987). Components of hostility and the severity of coronary artery disease. Psychosomatic Medicine, 49, 127-1359.

The Multicenter Postinfarction Research Group (1983). Risk stratification and survival after myocardial infarction. New England Fournal of Medicine, 309, 331-336.

Williams, R. B., Haney, T. L., Lee, K. L., Kong, Y.-H., Blumenthal, J. A. and Whalen, R. E. (1980). Type A behavior, hostility, and coronary atherosclerosis. Psychosomatic Medicine, 42, 539-549.

Williams, R. B., Barefoot, J. C., Haney, T. L., Harrell, F. E., Blumenthal, J. A., Pryor, D. B. and Peterson, B. (1988). Type A behavior and angiographically documented coronary atherosclerosis in a sample of 2289 patients. Psychosomatic Medicine, 50, 139-152.

Young, L D., Anderson, A. A., Barboriak, J. J. and Hoffman, R. G. (1980). Attitudinal and behavioral correlates of coronary heart disease. Fournal of Psychosomatic Research, 24, 311 318.

Zyzanski, S. J., Everist, M., Flessas, A., Jenkins, C. D. and Ryan, T. J. (1976). Psychological correlates of coronary angiographic findings. Archives of Internal Medicine, 136, 1234 1237. 


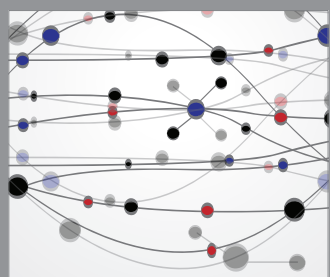

The Scientific World Journal
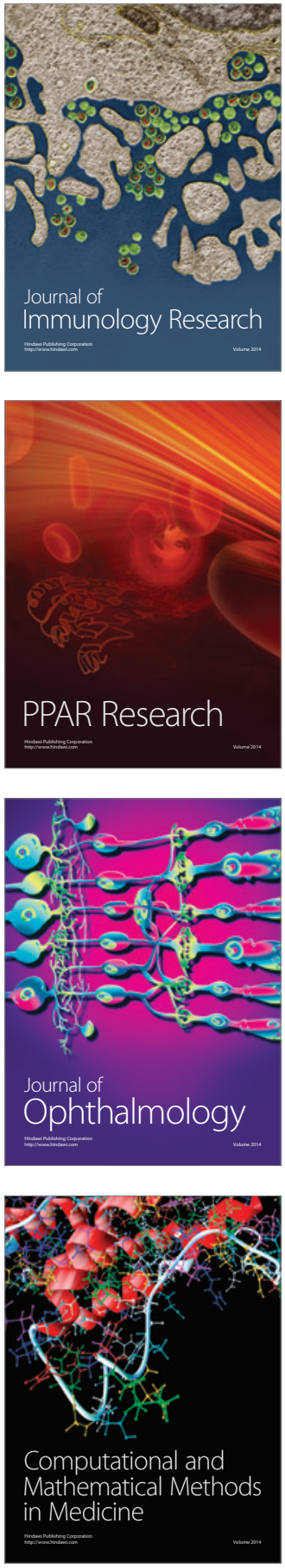

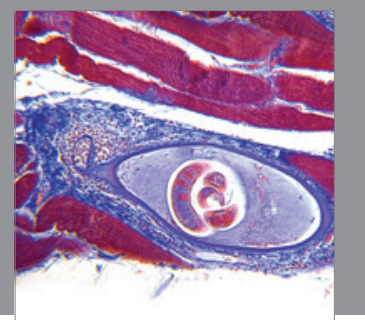

Gastroenterology

Research and Practice
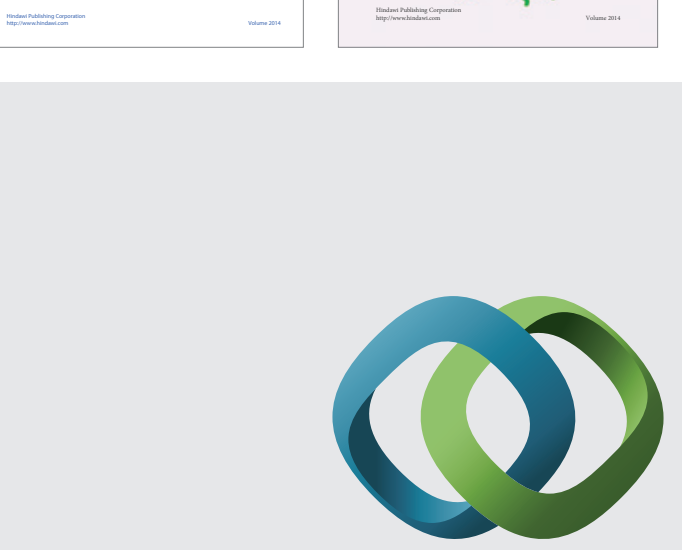

\section{Hindawi}

Submit your manuscripts at

http://www.hindawi.com
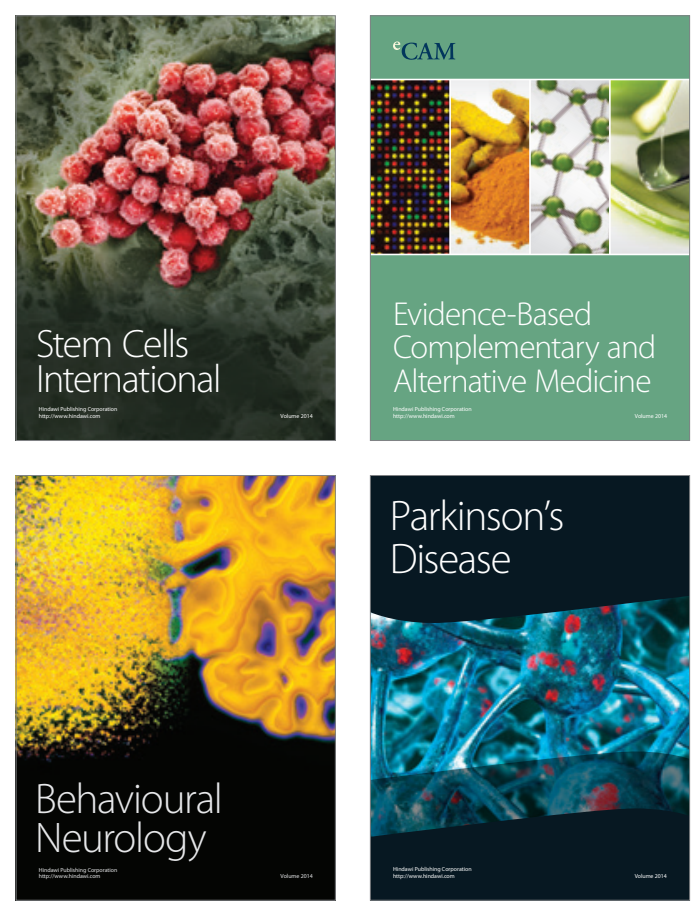

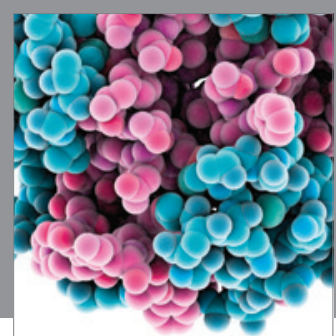

Journal of
Diabetes Research

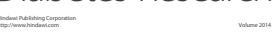

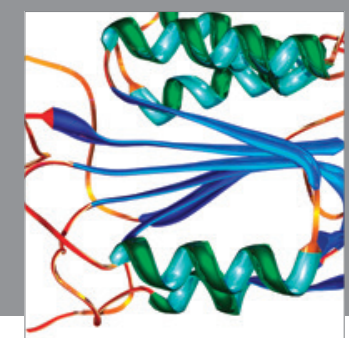

Disease Markers
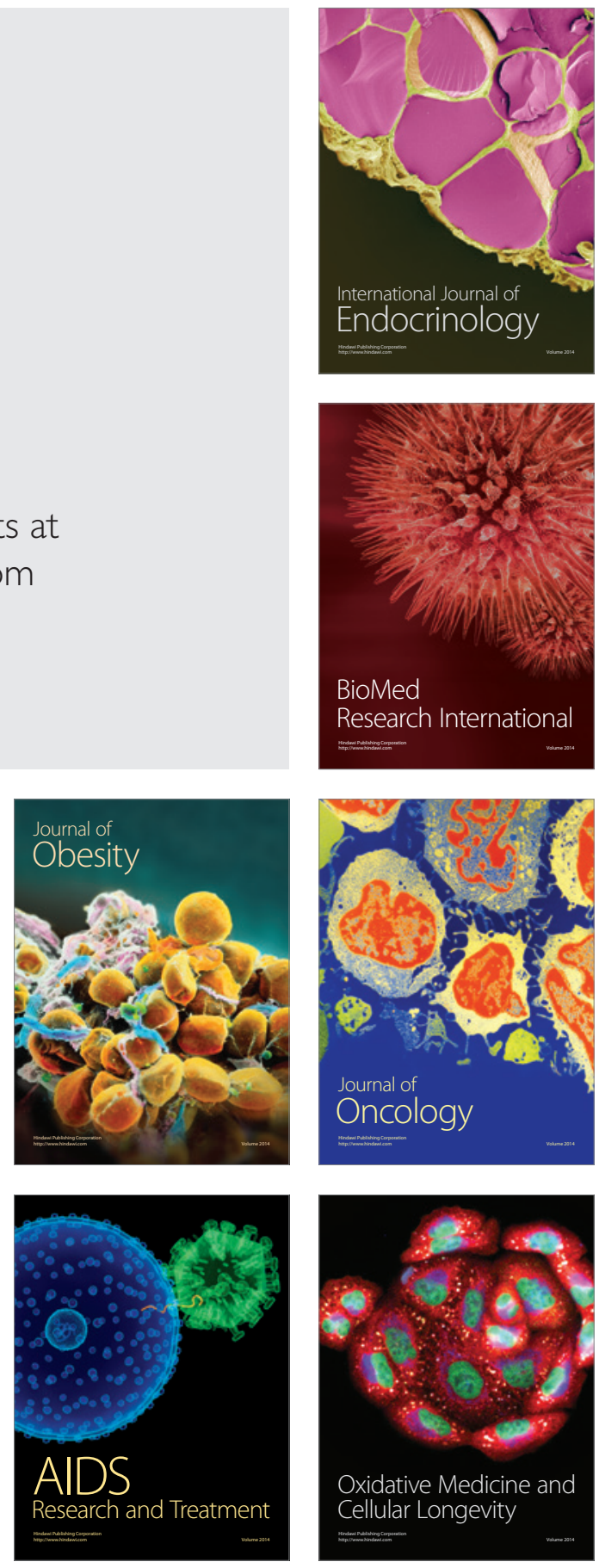